Erratum

Oper Orthop Traumatol 2020 · 32:531 https://doi.org/10.1007/s00064-020-00679-3 Online publiziert: 20. November 2020

(c) Springer Medizin Verlag GmbH, ein Teil von Springer Nature 2020
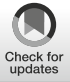

H. Kohlhof - M. Jaenisch · S. Koob - M. Friedrich - D. C. Wirtz

Klinik und Poliklinik für Orthopädie und Unfallchirurgie, Rheinische Friedrich-Wilhelms Universität Bonn, Bonn, Deutschland

\title{
Erratum zu: Distaler Femurersatz bei ausgedehnten femoralen Defekten in der Revisionsendoprothetik
}

\section{Erratum zu:}

Oper Orthop Traumatol 2020

https://doi.org/10.1007/s00064-020-

00667-7

Leider wurden bei dem ursprünglichen Beitrag die Namen der Autoren S. Koob und D. C. Wirtz nicht korrekt genannt. Des Weiteren zeigte die • Abb. 1 zur AORI(Anderson Orthopedic Research Institute)-Klassifikation nach Engh fälschlicherweise die tibialen anstatt der femoralen knöchernen Defekte.

Wir bitten, die Korrekturen der Autorennamen und die korrekte Abb. $1 \mathrm{zu}$ beachten, und entschuldigen uns für die aufgetretenen Fehler.

\section{Korrespondenzadresse}

Dr. H. Kohlhof, MHBA

Klinik und Poliklinik für Orthopädie und Unfallchirurgie, Rheinische Friedrich-Wilhelms Universität Bonn

Sigmund-Freud Str. 25, 53127 Bonn,

Deutschland

hendrik.kohlhof@ukbonn.de

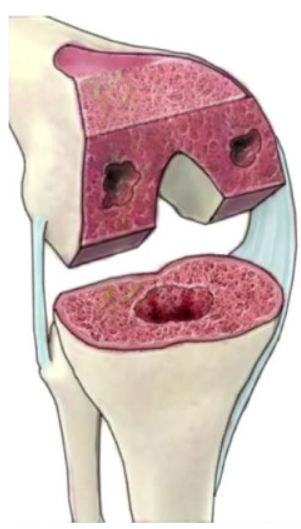

AORI Typ I

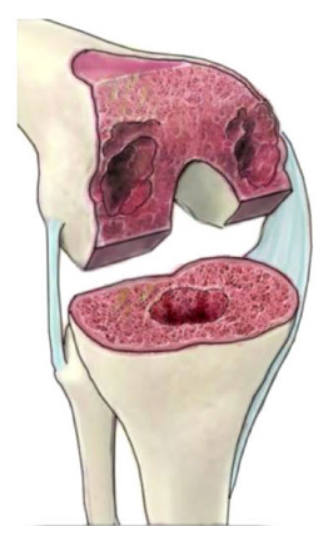

AORI Typ Ila

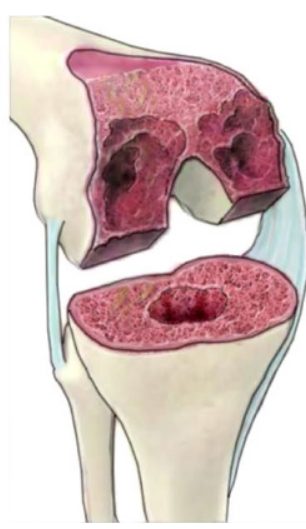

AORI Typ IIb

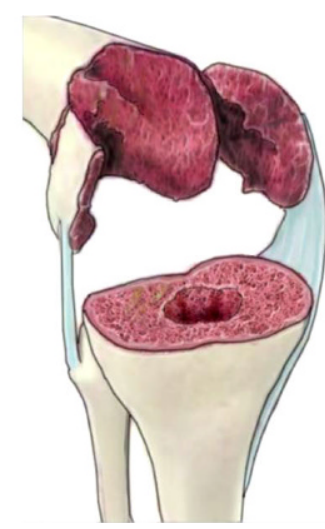

AORI Typ III
Abb. $1<$ AORI(Anderson Orthopedic Research Institute)-Klassifikation nach Engh

Die Online-Version des Originalartikels ist unter https://doi.org/10.1007/s00064-020-00667-7 zufinden. 